\title{
Supplement to: Optional Stopping with Bayes Factors: a categorization and extension of folklore results, with an application to invariant situations
}

\author{
A.A.Hendriksen, R. de Heide and P.D. Grünwald
}

\section{A Group theoretic preliminaries}

We start with some group-theoretical preliminaries; for more details, see e.g. Eaton, 1989, Wijsman, 1990, Andersson, 1982.

Definition 1 (Topological space). A non-empty set $S$ together with a fixed collection of subsets $\mathcal{T}$ is called a topological space $T=(S, \mathcal{T})$ if

(i) $S, \emptyset \in \mathcal{T}$,

(ii) $U \cap V \in \mathcal{T}$ for any two sets $U, V \in \mathcal{T}$, and

(iii) $S_{1} \cup S_{2} \in \mathcal{T}$ for any collections of sets $S_{1}, S_{2} \subseteq \mathcal{T}$.

The collection $\mathcal{T}$ is called a topology for $S$, and its members are called the open sets of $T$. A topological space $T$ is called Hausdorff if for any two distinct points $x, y \in T$ there exist disjoint open subsets $U, V$ of $T$ containing one point each.

Definition 2 ((Local) compactness). A topological space $T$ is compact if every open cover, that is, every collection $\mathcal{C}$ of open sets of $T$

$$
T=\bigcup_{U \in \mathcal{C}} U
$$

has a finite subcover: a finite subcollection $\mathcal{F} \in \mathcal{C}$ such that

$$
T=\bigcup_{V \in \mathcal{F}} V
$$

It is locally compact if for every $x \in T$ there exist an open set $U$ such that $x \in U$ and the closure of $U$, denoted by $C l(U)$, is compact, that is, the union of $U$ and all its limit points in $T$ is compact. We can also formulate this as each $x$ having a neighbourhood $U$ such that $C l(U)$ is compact.

Example 1 (Locally compact Hausdorff spaces). The reals $\mathbb{R}$ and the Euclidean spaces $\mathbb{R}^{n}$ together with the Euclidean topology (also called the usual topology) are locally compact Hausdorff spaces. $\mathbb{R}^{n}$ (for $n \in \mathbb{N}$ ) is locally compact because any open ball $B(x, r)$ has a compact closure $C l(B(x, r))=\left\{y \in \mathbb{R}^{n} ; d(x, y) \leq \epsilon\right\}$, where $d(x, y)$ is the Euclidean metric. Any discrete space is locally compact and Hausdorff as well, as any singleton is a neighbourhood that equals its closure, and it is compact only if it is finite. Infinite dimensional Banach spaces (function spaces) are for example not locally compact. 
Definition 3 (Group). A set $G$ together with a binary operation $\circ$, often called the group law, is called a group when

(i) there exists an identity element $e \in G$ for the group law $\circ$,

(ii) for every three elements $a, b, c \in G$, we have $(a \circ b) \circ c=a \circ(b \circ c)$ (associativity), and

(iii) for each element $a \in G$, there exists an inverse element, $a^{\dagger} \in G$, with $a \circ a^{\dagger}=a^{\dagger} \circ a=e$.

Transformation groups A group that consists of a set $G$ of transformations on some set $S$ is called a transformation group. We also say that the group $G$ acts on the set $S$. A transformation is a mapping from $S$ to itself that preserves certain properties, such as isometries in the Euclidean plane. Transformation groups are usually not commutative, that is $a \circ b \neq b \circ a$ for $a, b \in G$.

Definition 4 (Topological group). A topological space $G$ that is also a group is called a topological group when the group operation $\circ$ is continuous, that is, for $a, b \in G$, we have that the operations of product

(i) $G \times G \rightarrow G:(a, b) \mapsto a \circ b$, and taking the inverse

(ii) $G \rightarrow G: a \mapsto a^{\dagger}$,

are continuous, where $G \times G$ has the product topology.

A topological group for which the underlying topology is locally compact and Hausdorff, is called a locally compact group.

Definition 5 (Eaton [1989], Definition 2.1). Let $Y$ be a set, and let $G$ be a group with identity element e. A function $F: Y \times G \rightarrow Y$ satisfying

1. $F(y, e)=y, y \in Y$

2. $F\left(y, g_{1} g_{2}\right)=F\left(F\left(y, g_{1}\right), g_{2}\right), g_{1}, g_{2} \in G, y \in Y$

specifies $G$ acting on the right of $Y$.

In practice, $F$ is omitted: we will write $y \cdot g$ for a group element $g$ acting on the right of $y \in Y$. For a subset $A \subseteq Y$, we write $A \cdot g:=\{a \cdot g \mid a \in A\}$.

Right Haar measure

Definition 6 (Conway 2013, Example 1.11). Let $G$ be a locally compact topological group. Then the right invariant Haar measure (in short: right Haar measure) for $G$ is a Borel measure $\nu$ satisfying

1. $\nu(A)>0$ for every nonempty open set $A \subseteq G$,

2. $\nu(K)<\infty$ for every compact set $K \subseteq G$,

3. $\nu(A \cdot g)=\nu(A)$ for every $g \in G$ and every measurable $A \subseteq G$. 


\section{B Proofs Omitted from Main Text}

Proof. [of Lemma 1] Let $A \subset \mathbb{R}_{>0}$ be any Borel measurable set. In the equations below, the sum and integral can be swapped due to the monotone convergence theorem and the fact that $B_{\tau}$ is a positive function.

$$
\begin{aligned}
& \int_{A} \mathrm{~d} P_{1}^{\left[B_{\tau}\right]}=\int_{\Omega} \mathbb{1}_{\left\{B_{\tau} \in A\right\}} \mathrm{d} P_{1}^{\left[B_{\tau}\right]} \\
& =\sum_{n=0}^{\infty} \int_{\left\langle\mathcal{X}^{n}\right\rangle} \mathbb{1}_{\left\{B_{\tau} \in A\right\}} \mathbb{1}_{\{\tau=n\}} \mathrm{d} P_{1}^{(n)} \\
& \stackrel{(3)}{=} \sum_{n=0}^{\infty} \int_{\left\langle\mathcal{X}^{n}\right\rangle} \mathbb{1}_{\left\{B_{n} \in A\right\}} \mathbb{1}_{\{\tau=n\}} P_{1}^{(n)}(\tau=n) \cdot \mathrm{d} P_{1}^{(n)}(\cdot \mid \tau=n) \\
& =\sum_{n=0}^{\infty} \int_{\left\langle\mathcal{X}^{n}\right\rangle} \mathbb{1}_{\left\{B_{n} \in A\right\}} P_{1}^{(n)}(\tau=n) \cdot \mathrm{d} P_{1}^{(n)}(\cdot \mid \tau=n) \\
& \stackrel{(5)}{=} \sum_{n=0}^{\infty} \int_{r>0} \mathbb{1}_{\{r \in A\}} P_{1}^{(n)}(\tau=n) \cdot \mathrm{d} P_{1}^{\left[B_{n}\right]}(\cdot \mid \tau=n) \\
& =\sum_{n=0}^{\infty} \int_{r>0} \mathbb{1}_{\{r \in A\}} \frac{\mathrm{d} P_{1}{ }^{\left[B_{n}\right]}(\cdot \mid \tau=n)}{\mathrm{d} P_{0}{ }^{\left[B_{n}\right]}(\cdot \mid \tau=n)}(r) P_{1}^{(n)}(\tau=n) \cdot \mathrm{d} P_{0}{ }^{\left[B_{n}\right]}(\cdot \mid \tau=n) \\
& \stackrel{(*)}{=} \sum_{n=0}^{\infty} \int_{r>0} \mathbb{1}_{\{r \in A\}} \frac{P_{0}^{(n)}(\tau=n)}{P_{1}^{(n)}(\tau=n)} \cdot r \cdot P_{1}^{(n)}(\tau=n) \cdot \mathrm{d} P_{0}{ }^{\left[B_{n}\right]}(\cdot \mid \tau=n) \\
& =\sum_{n=0}^{\infty} \int_{r>0} \mathbb{1}_{\{r \in A\}} r P_{0}^{(n)}(\tau=n) \cdot \mathrm{d} P_{0}{ }^{\left[B_{n}\right]}(\cdot \mid \tau=n) \\
& =\sum_{n=0}^{\infty} \int_{\left\langle\mathcal{X}^{n}\right\rangle} \mathbb{1}_{\left\{B_{n} \in A\right\}} \cdot B_{n} \cdot P_{0}^{(n)}(\tau=n) \cdot \mathrm{d} P_{0}^{(n)}(\cdot \mid \tau=n) \\
& =\sum_{n=0}^{\infty} \int_{\left\langle\mathcal{X}^{n}\right\rangle} \mathbb{1}_{\left\{B_{n} \in A\right\}} \mathbb{1}_{\{\tau=n\}} \cdot B_{n} \cdot P_{0}^{(n)}(\tau=n) \cdot \mathrm{d} P_{0}^{(n)}(\cdot \mid \tau=n) \\
& =\sum_{n=0}^{\infty} \int_{\left\langle\mathcal{X}^{n}\right\rangle} \mathbb{1}_{\left\{B_{\tau} \in A\right\}} \mathbb{1}_{\{\tau=n\}} \cdot B_{n} \mathrm{~d} P_{0}^{(n)} \\
& =\int_{\Omega} \mathbb{1}_{\left\{B_{\tau} \in A\right\}}\left(\sum_{n=0}^{\infty} \mathbb{1}_{\{\tau=n\}} B_{n}\right) \mathrm{d} P_{0} \\
& =\int_{\Omega} \mathbb{1}_{\left\{B_{\tau} \in A\right\}} B_{\tau} \mathrm{d} P_{0} \\
& \stackrel{(14)}{=} \int_{A} t P_{0}^{\left[B_{\tau}\right]}(d t) \text {, }
\end{aligned}
$$

where $(*)$ follows because of our fixed $n$-calibration assumption. Furthermore, $(3)$ follows from the following equality for any $C \in \mathcal{F}$

$$
P_{1}^{(n)}(C \cap\{\tau=n\})=P_{1}^{(n)}(\tau=n) \cdot P_{1}^{(n)}(C \mid \tau=n),
$$


and in (5) we perform a change of variables where we integrate over the possible values of the Bayes Factor instead of over the outcome space, which we repeat in (14).

We have shown that the function $g$ defined by $g(t)=t$ is the Radon-Nikodym derivative $\frac{\left.\mathrm{d} P_{1}^{[B \tau}\right]}{\mathrm{d} P_{0}\left[B_{\tau}\right]}$.

Proof. [of Lemma 2] Let $A$ be any Borel subset of $\mathbb{R}_{>0}$. We have:

$$
\begin{aligned}
\int_{A} \mathrm{~d} \bar{P}_{1}^{\left[\gamma_{n}\right]}\left(\cdot \mid x^{m}, \tau=n\right) & =\int_{\left\langle\mathcal{X}^{n}\right\rangle} \mathbb{1}_{\left\{\gamma_{n} \in A\right\}} \mathrm{d} \bar{P}_{n}^{(1)}\left(\cdot \mid x^{m}, \tau=n\right) \\
& =\int_{\left\langle\mathcal{X}^{n}\right\rangle} \mathbb{1}_{\left\{\gamma_{n} \in A\right\}}\left(\frac{\mathrm{d} \bar{P}_{n}^{(1)}\left(\cdot \mid x^{m}, \tau=n\right)}{\mathrm{d} \bar{P}_{n}^{(0)}\left(\cdot \mid x^{m}, \tau=n\right)}\right) \mathrm{d} \bar{P}_{n}^{(0)}\left(\cdot \mid x^{m}, \tau=n\right) \\
& \stackrel{(*)}{=} \int_{\left\langle\mathcal{X}^{n}\right\rangle} \mathbb{1}_{\left\{\gamma_{n} \in A\right\}} \gamma_{n} \cdot\left(\frac{\pi\left(H_{0} \mid x^{m}, \tau=n\right)}{\pi\left(H_{1} \mid x^{m}, \tau=n\right)}\right) \mathrm{d} \bar{P}_{n}^{(0)}\left(\cdot \mid x^{m}, \tau=n\right) \\
& =\int_{\left\langle\mathcal{X}^{n}\right\rangle} \mathbb{1}_{\left\{\gamma_{n} \in A\right\}} \gamma_{n}\left(\frac{\bar{P}_{0}\left(\tau=n \mid x^{m}\right) \pi\left(H_{0}\right)}{\bar{P}_{1}\left(\tau=n \mid x^{m}\right) \pi\left(H_{1}\right)}\right) \mathrm{d} \bar{P}_{n}^{(0)}\left(\cdot \mid x^{m}, \tau=n\right) \\
& =\left(\frac{\bar{P}_{0}\left(\tau=n \mid x^{m}\right) \pi\left(H_{0} \mid x^{m}\right)}{\bar{P}_{1}\left(\tau=n \mid x^{m}\right) \pi\left(H_{1} \mid x^{m}\right)}\right) \cdot \int_{A} \gamma_{n} \mathrm{~d} \bar{P}_{0}^{\left[\gamma_{n}\right]}\left(\cdot \mid x^{m}, \tau=n\right),
\end{aligned}
$$

where, for the case $m=0,(*)$ follows from (3), which can be verified to be still valid in our generalized setting. The case $m>0$ follows in exactly the same way, by shifting the data by $m$ places (so that the new $x_{1}$ becomes what was $x_{m+1}$, and treating, for $k=0,1, \pi\left(H_{k} \mid x^{m}\right.$ ) as the priors for this shifted data problem, and then applying the above with $m=0$ ).

We have shown that the Radon-Nikodym derivative $\frac{\mathrm{d} \bar{P}_{1}^{[\gamma n]}\left(\cdot \mid x^{m}\right)}{\mathrm{d} \bar{P}_{0}^{[\gamma n]}\left(\cdot \mid x^{m}\right)}$ at $\gamma_{n}$ is given by $\gamma_{n}$. $\frac{\bar{P}_{0}\left(\tau=n \mid x^{m}\right) \pi\left(H_{1} \mid x^{m}\right)}{\bar{P}_{1}\left(\tau=n \mid x^{m}\right) \pi\left(H_{0} \mid x^{m}\right)}$, which is what we had to show.

Proof. [of Lemma 7] Let $A^{\prime}$ denote the event $V_{n}=1$ and let $A \subset \mathbb{R}_{>0}$ be a Borel measurable subset of the positive real numbers. We have that $\beta_{n}$ is a function of the maximal invariant $U_{n}$ as defined in Definition 3, and we write $\beta_{n}\left(U_{n}\right)$. With this notation, we have:

$$
\begin{aligned}
& P_{1, g}^{\left[\beta_{n}\right]}\left(A \mid A^{\prime}\right)=\int_{\mathbb{R}_{>0}} \mathbb{1}_{\{A\}} \mathrm{d} P_{1, g}^{\left[\beta_{n}\right]}\left(\cdot \mid A^{\prime}\right) \\
& \stackrel{(2)}{=} \int_{\mathcal{U}_{n}} \mathbb{1}_{\left\{\beta_{n}\left(U_{n}\right) \in A\right\}} \mathrm{d} P_{1, g}^{\left[U_{n}\right]}\left(\cdot \mid A^{\prime}\right) \\
& =\int_{\mathcal{U}_{n}} \mathbb{1}_{\left\{\beta_{n}\left(U_{n}\right) \in A\right\}} \frac{\mathrm{d} P_{1, g}^{\left[U_{n}\right]}\left(\cdot \mid A^{\prime}\right)}{\mathrm{d} P_{0, g}^{\left[U_{n}\right]}\left(\cdot \mid A^{\prime}\right)} \mathrm{d} P_{0, g}^{\left[U_{n}\right]}\left(\cdot \mid A^{\prime}\right) \\
& \stackrel{(4)}{=} \int_{\mathcal{U}_{n}} \mathbb{1}_{\left\{\beta_{n}\left(U^{n}\right) \in A\right\}} \frac{P_{0, g}^{(n)}\left(A^{\prime}\right)}{P_{1, g}^{(n)}\left(A^{\prime}\right)} \frac{\mathrm{d} P_{1, g}^{\left[U_{n}\right]}}{\mathrm{d} P_{0, g}^{\left[U_{n}\right]}} \mathrm{d} P_{0, g}^{\left[U_{n}\right]}\left(\cdot \mid A^{\prime}\right) \\
& \stackrel{(5)}{=} \frac{P_{0, g}^{(n)}\left(A^{\prime}\right)}{P_{1, g}^{(n)}\left(A^{\prime}\right)} \cdot \int_{\mathcal{U}_{n}} \mathbb{1}_{\left\{\beta_{n}\left(U^{n}\right) \in A\right\}} \beta_{n}\left(U_{n}\right) \mathrm{d} P_{0, g}^{\left[U_{n}\right]}\left(\cdot \mid A^{\prime}\right) \\
& =\frac{P_{0, g}^{(n)}\left(A^{\prime}\right)}{P_{1, g}^{(n)}\left(A^{\prime}\right)} \cdot \int_{\mathbb{R}_{>0}} \mathbb{1}_{\{A\}} t \mathrm{~d} P_{0, g}^{\left[\beta_{n}\right]} A^{\prime}(t),
\end{aligned}
$$


where step (2) holds because $\beta_{n}$ is $\mathcal{G}_{n}$-measurable. On the set $A^{\prime}$ we have

$$
\frac{\mathrm{d} P_{1, g}^{\left[U_{n}\right]}\left(\cdot \mid A^{\prime}\right)}{\mathrm{d} P_{0, g}^{\left[U_{n}\right]}\left(\cdot \mid A^{\prime}\right)} \frac{P_{1, g}^{(n)}\left(A^{\prime}\right)}{P_{0, g}^{(n)}\left(A^{\prime}\right)}=\frac{\mathrm{d} P_{1, g}^{\left[U_{n}\right]}}{\mathrm{d} P_{0, g}^{\left[U_{n}\right]}},
$$

which explains step (4), and step (5) follows from the definition of $\beta_{n}$ in Equation (29).

We have shown that $\frac{P_{0, g}^{(n)}\left(A^{\prime}\right)}{P_{1, g}^{(n)}\left(A^{\prime}\right)} \cdot t$ is equal to the Radon-Nikodym derivative $\frac{\mathrm{d} P_{1, g}^{\left[\beta_{n}\right]}\left(\cdot \mid V_{n}=1\right)}{\mathrm{d} P_{0, g}^{[\beta]}\left(\cdot \mid V_{n}=1\right)}$, which is what we had to prove.

\section{References}

S. Andersson. Distributions of maximal invariants using quotient measures. The Annals of Statistics, 10(3):955-961, 1982. ISSN 00905364.

J. B. Conway. A course in functional analysis, volume 96. Springer Science \& Business Media, 2013.

M. L. Eaton. Group invariance applications in statistics. Regional Conference Series in Probability and Statistics, 1:i-133, 1989. ISSN 19355912.

R. A. Wijsman. Invariant measures on groups and their use in statistics. Institute of Mathematical Statistics, 1990. ISBN 9780940600195. 